\title{
BENEFICIOS DE LOS JUEGOS DE PREGUNTAS COMO ESTRATEGIA DIDÁCTICA PARA LA ENSEÑANZA DE LA BIOLOGÍA
}

\author{
Benefits of Quiz Games as a Teaching \\ Strategy in the Teaching of Biology
}

\section{Benefícios dos jogos de perguntas como estratégia didática para o ensino da biologia}

\author{
Yurieth García Montes ${ }^{1}$
}

Fecha de recepción: 4 de agosto de 2017

Fecha de aceptación: $1 .^{\circ}$ de marzo de 2018

\section{Resumen}

Esta investigación buscó determinar cuáles son los beneficios de los juegos de preguntas como estrategia didáctica en el proceso de enseñanza-aprendizaje de la biología, en estudiantes de séptimo grado, de una institución educativa privada ubicada en la vía Suba-Cota, en 2016. Los juegos empleados fueron convencionales y se ajustaron para plantear preguntas al finalizar las temáticas. Su efectividad se estableció con una sustentación antes y una después de su implementación. El criterio de avance se definió con el diseño e implementación de una escala valorativa según el número de aciertos en las preguntas. Asimismo, para conocer el beneficio de los juegos de preguntas, se evaluó la percepción de los estudiantes frente a su uso, siguiendo una metodología mixta de tipo cualitativo y cuantitativo, con un enfoque interpretativo. En el análisis de las encuestas, se utilizó el método descriptivointerpretativo y se organizó la información en categorías, según la grounded theory, o método comparativo constante (MCC). Los resultados y análisis de la estrategia didáctica implementada muestran que los juegos de preguntas son un elemento motivador que fomenta la creatividad, el interés y la responsabilidad del estudiante con su proceso de formación. Además, se resalta que a través del juego se pueden introducir y reforzar elementos teóricos que posibilitan la comprensión de los conceptos de la biología, ya que pueden enfocar la atención y posiblemente mejorar la memoria.

Palabras clave: Juego, aprendizaje significativo, motivación, estrategia didáctica

1 Especialista en Planeación Ambiental y Manejo Integral de los Recursos Naturales de la Universidad Militar Nueva Granada y licenciada en Biología de la Universidad Pedagógica Nacional. Correo electrónico: matzgm@yahoo.es. 


\section{Abstract}

This research sought to determine the benefits of question games as a didactic strategy in the teaching-learning process of biology, in seventh grade students, of a private educational institution located on the Suba-Cota road, in 2016. The games used were conventional and adjusted to pose questions at the end of the topics. Its effectiveness was established with an oral defense before and after its implementation. The criterion of progress was defined with the design and implementation of a rating scale according to the number of correct answers in the questions. Likewise, in order to know the benefit of the question games, the perception of the students regarding their use was evaluated, following a mixed methodology of qualitative and quantitative type, with an interpretive approach. In the analysis of the surveys, the descriptive-interpretative method was used and the information was organized into categories, according to the grounded theory, or constant comparative method $(\mathrm{ccm})$. The results and analysis of the didactic strategy implemented show that question games are a motivating element that encourages creativity, interest and responsibility of the student with his educational process. In addition, it is emphasized that through the games can be introduced and reinforced theoretical elements that enable the understanding of the concepts of biology, as they can focus the attention and possibly improve memory.

Key words: play; meaningful learning; motivation; didactic strategy

\section{Resumo}

Este estudo procurou determinar quais são os benefícios de jogos de perguntas como estratégia didática no processo de ensinoaprendizagem da biologia em alunos do sétimo de uma instituição privada de ensino localizada na rota Suba-Cota, em 2016. Os jogos utilizados foram convencionais e foram ajustados para colocar questões no final dos tópicos. Sua eficácia foi estabelecida com uma sustentação antes e depois de sua implementação. O critério de progresso foi definido com o desenho e implementação de uma escala de classificação de acordo com o número de respostas corretas nas questões. Além disso, para saber o benefício dos jogos de perguntas, a percepção dos estudantes quanto ao seu uso foi avaliada, seguindo uma metodologia mista de tipo qualitativa e quantitativa, com uma abordagem interpretativa. Na análise dos inquéritos, utilizou-se o método descritivo-interpretativo e as informações foram organizadas em categorias, de acordo com a teoria fundamentada, ou método comparativo constante (mcc). Os resultados e a análise da estratégia didática implementada mostram que os conjuntos de perguntas são um elemento motivador que estimula a criatividade, o interesse e a responsabilidade do aluno com seu processo de formação. Além disso, destaca-se que através do jogo podem ser introduzidos e reforçados elementos teóricos que permitem a compreensão dos conceitos de biologia, porque eles podem concentrar a atenção e, possivelmente, melhorar a memória.

Palavras-chave: jogo; aprendizagem significativa; motivação; estratégia didática 
El juego es la expresión de nuestra creatividad, y la creatividad, en mi opinión, está en la raíz misma de nuestra capacidad de aprender, de lidiar, y de llegar a ser lo que podemos ser.

Fred Rogers

\section{Introducción}

Durante algún tiempo el conocimiento de la biología se ha contemplado y transmitido como una colección de hechos, principios, leyes, reglas e interacciones lógicas (Stenhouse, 1987). Sin embargo, esto no es suficiente para generar un conocimiento significativo, es decir, cuando el estudiante tiene la posibilidad de vincular de manera estable y clara sus conocimientos previos con la nueva información y, a partir de allí, elaborar una reestructuración y asimilación de estos.

Por otra parte, no favorece el desarrollo de las diferentes potencialidades de los estudiantes, esto es, los planos cognoscitivo (saber), afectivo (ser) y comportamental (saber hacer). En ese sentido, Estebaranz (1994), Fernández (1974), Escudero (1981), Titone (1976), Díaz (1999) y Mallart (2001), entre otros, mencionan que la didáctica es de vital importancia en el proceso de enseñanza-aprendizaje, ya que permite buscar e implementar métodos y estrategias eficaces, relacionados no solo con los contenidos, sino con las dificultades para aprenderlos.

Al respecto, estas actividades son relevantes en las ciencias naturales, porque la mayor parte de sus teorías no se basan en leyes sino en conceptos (Mayr, 2006) y es allí donde se presentan los obstáculos, por lo abstracto y complejo de algunos de sus temas (Stewart, 1983). Además, emplear la didáctica contribuye a generar mayor motivación en los estudiantes por aprender. De hecho, Cook y Artino (2016) relacionan la motivación con la expectativa de éxito; así se genera la convicción de lograr la tarea asignada. Asimismo, como lo indican Ryan y Deci (2000) facilita que se involucren todo el tiempo.

En cuanto a la motivación y a las creencias que surgen de ella, predomina la percepción porque tiene la función de realizar abstracciones a través de las cualidades que definen lo esencial de la realidad externa. En otras palabras, la percepción permite constituir las representaciones mentales (Oviedo, 2004); de ahí que posibilite reformular las experiencias y las estructuras perceptuales, y como lo señala Goldstone (1998) este aprendizaje perceptivo, favorece articular la información recogida con el uso que se le va a dar.

De esta manera, el interés, la motivación, y la percepción están estrechamente relacionadas con la didáctica, ya que permiten optimizar el proceso de enseñanza- aprendizaje.
Además, es indudable que influyen en la actitud con la que los estudiantes asumen las actividades (Reeve, 1996), y como las actitudes son las que permiten evaluar los objetos o sujetos dependiendo de las creencias disponibles, conducen a actuar de modo favorable o desfavorable hacia estos (Gargallo, Pérez, Serra, Sánchezy Ros, 2007), por tanto, son fundamentales en los resultados escolares.

Lo anterior se evidencia en el gran número de investigaciones que han resaltado su importancia en la construcción de un aprendizaje significativo, al permitir que los estudiantes sientan confianza en sí mismos y seguridad al participar (Afanador y Mosquera, 2017; Alonso, 1998; Becerra, Valderrama y Torres, 2014; González y Maytorena, 2001; Jiménez y Hernández, 2001; Muñoz de Bustillo, Hernández y García, 1998).

En cuanto al juego, autores como Piaget (1971) y Erikson (1972) resaltan su importancia en el desarrollo infantil. Por su parte, Winnicott (1971) lo relaciona con la construcción del ser humano, y Klein (1929) expresa que es el mejor medio de expresión del niño. En ese sentido, Wallon (1942) indica que facilita el acceso y dominio del campo simbólico, por lo tanto, es claro que el juego propicia espacios de desarrollo, donde se reconoce la importancia de las reglas. A la par, Cagigal (1971), Gruppe (1976), Moor (1981) y Blanchard y Chesca (1986) lo definen como un elemento fundamental en la educación, que favorece la cohesión y la solidaridad como grupo.

Por lo que respecta a su clasificación, esta puede ser bastante amplia; sin embargo, Caillois (1986) presenta cuatro secciones: la competencia (Agón), el azar (Alea), el simulacro (Mimicry) o el vértigo (Ilinx). De esta forma, enmarcados dentro de la competencia, se puede hablar de juegos de preguntas, ya que el autor comenta que en esta categoría hay una rivalidad con igualdad de oportunidades entre límites definidos, como puede ser la memoria. Así, los juegos de preguntas son una serie de interrogantes que se plantean en el marco de un concurso o juego, donde todos tienen las mismas posibilidades de participar, porque las respuestas se vinculan a un conocimiento compartido de manera general.

Tras esta revisión, el juego no se ve solo como una actividad de ocio y esparcimiento, sino como un espacio placentero de construcción, que le permite al niño desarrollar los aspectos afectivo, cognitivo y social, para conocer y participar en el entorno en el que se encuentra. De hecho, el juego se ha empleado en la educación para superar algunos obstáculos en temas o conceptos concretos en el aprendizaje de la biología; un ejemplo de ello son las investigaciones de Burton y Dobson (2009), Latham y Scully (2008); Mengascini y Menegaz (2005), y Santisteban (1990).

(1)


De igual manera, los trabajos de Meloy Hernández (2014); Orozco y Perdomo (2015); Melo, (2017); Sanabria, Sandoval y Arango (2017) evidencian las posibilidades del juego en la enseñanza de las ciencias naturales.

A su vez, el juego desempeña un rol importante en el aprendizaje y la memoria. Por ejemplo Deveau, Jaeggi, Zordan, Phung y Seitz (2014) revelan que los juegos se pueden utilizar para crear ambientes atractivos para el aprendizaje, enfocados en una memoria de trabajo, es decir, un sistema con la capacidad de almacenar y manipular la información en un momento dado. Igualmente, Kafai y Burke (2015), basados en el constructivismo, sugieren las diferentes dimensiones de los juegos en el aprendizaje.

A partir de allí, el objetivo de este trabajo es determinar cuáles son los beneficios de los juegos de preguntas en el proceso de enseñanza-aprendizaje de la biología, en estudiantes de séptimo grado de una institución educativa privada. Este planteamiento contempla la necesidad de ver los juegos como una forma de generar interés en los estudiantes por el proceso de enseñanza- aprendizaje. Asimismo, genera una oportunidad para los docentes, al producir actividades que dinamicen las clases y resulten agradables y significativas para los estudiantes.

\section{Metodología}

El trabajo se llevó a cabo en una institución educativa privada, en la vía Suba-Cota (Bogotá) en 2016. Participaron 41 estudiantes de séptimo grado (cursos A y B). Con un rango de edad entre 13 y 15 años. Las propuestas de juego se realizaron a lo largo de las clases semanales de ciencias.

La base de la indagación fue la investigación mixta, ya que representa el más alto grado de integración o combinación entre los enfoques cualitativo y cuantitativo (Hernández, Fernández y Baptista, 2003). Además, porque no es posible referir lo cuantitativo sin hablar de lo cualitativo (Cerda, 2002).

El proceso se realizó en cinco fases.

\section{Primera fase: presentación de la temática}

Inicialmente, se les informó a los estudiantes sobre el tema que se iba a trabajar y sus objetivos, ya que esto les da una sensación de propiedad y autoría lo cual favorece que se involucren en el aprendizaje (Newman, 1992). Al finalizar la temática, se realizaba una sustentación. A continuación, se explican los temas abordados.

\section{Flujo de materia y energía}

Se considera que todos los organismos necesitan materia y energía para llevar a cabo todas sus funciones vitales. La energía utilizada proviene del sol y cuando los organismos la consumen, esta ya no queda disponible para otros. En cuanto a la materia, los organismos la transforman constantemente.

\section{Ciclos biogeoquímicos}

Teniendo en cuenta que la materia circula desde el mundo vivo hacia el ambiente abiótico y de regreso, esa circulación constituye los ciclos biogeoquímicos. Estos son procesos naturales que permiten el flujo de los elementos desde el medio ambiente hacia los organismos.

\section{Niveles de organización de los ecosistemas}

Los diferentes niveles de organización han sido considerados por Odum (1971) como sistemas biológicos, cada uno con complejidades e interacciones. Dentro de los niveles se abordaron especie, población, comunidad y ecosistema.

\section{Cadenas y redes tróficas}

Considerando que el conjunto de seres vivos de un ecosistema obtiene la materia y la energía de un modo semejante se denomina nivel trófico. Estos se representan de manera lineal (cadenas) o en redes, una vez se establecen las conexiones entre una y otra cadena.

\section{Segunda fase: puesta en marcha de los juegos de preguntas}

En la segunda fase, después de la sustentación, se realizaron juegos de preguntas, considerando que la elaboración de preguntas y la búsqueda de respuestas a ellas son fundamentales en el desarrollo científico. Estas no solo determinan el método de investigación y el conocimiento generado, sino también son importantes en el proceso de enseñanza-aprendizaje de las ciencias, al movilizar las concepciones del estudiante, como lo menciona Giordan (1989).

Los juegos utilizados fueron convencionales y se ajustaron para plantear las preguntas. Estos se detallan a continuación. 
Como ejemplo de las preguntas utilizadas se pueden citar:

Actividad utilizada en las temáticas flujo de materia y energía, y ciclos biogeoquímicos.

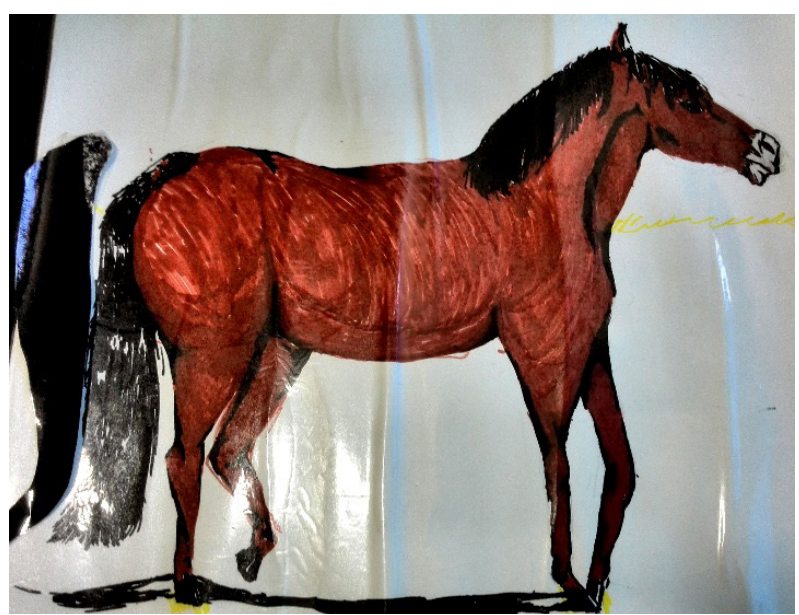

Realizado por una estudiantes del grupo A

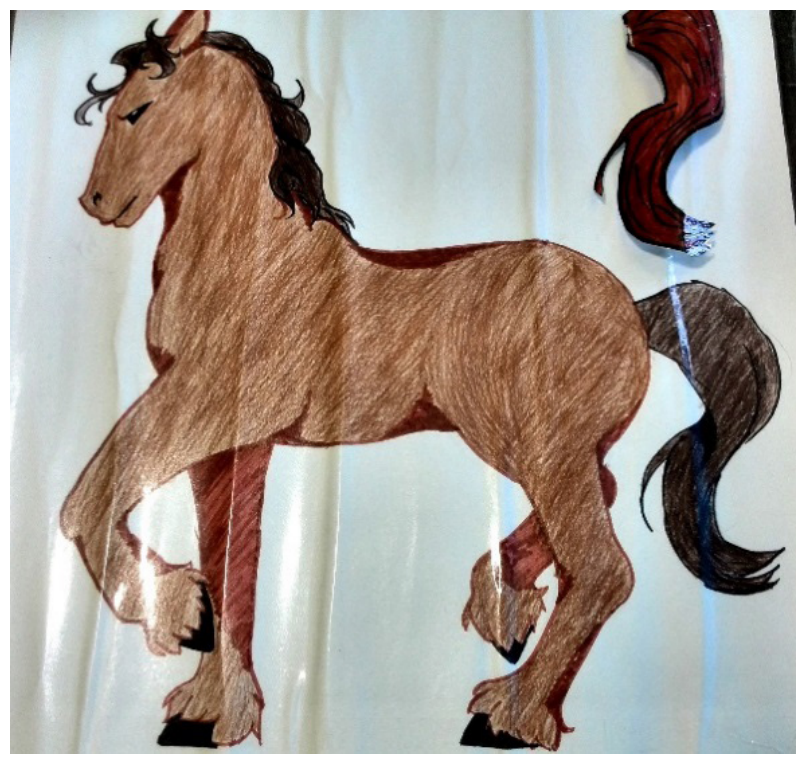

Realizado por una estudiantes del grupo B

Fotografía 1. Ponerle la cola al caballo

En este juego (fotografía 1), el grupo de estudiantes se dividió en dos y se organizó en filas, de manera que el primer estudiante tenía los ojos vendados y ubicaba la cola del caballo. Si lo hacía bien y respondía la pregunta correctamente tenía un total de 3 puntos, si no ubicaba la cola en el sitio correcto pero respondía bien la pregunta, obtenía dos puntos. Si no se respondía la pregunta no había puntos. El grupo que ganaba era el que mayor puntaje tenía al final.

- ¿La energía que utiliza un organismo puede ser a su vez utilizada por otro?

- ¿La energía utilizada por los organismos tiene un flujo unidireccional, o bidireccional?

- ¿La materia tiene un flujo unidireccional, o bidireccional?

- ¿Cuál es la principal fuente de energía para la Tierra?

- ¿Cuál es el porcentaje de energía del sol que perciben los consumidores primarios?

- ¿Cuál es el proceso utilizado por los vegetales para captar el carbono?

- ¿Cuál es la mayor reserva de fósforo en la Tierra?

- El carbono es el primer y principal elemento de la estructura de los seres vivos. ¿Verdadero o falso?

- La mayoría de los organismos puede utilizar directamente el nitrógeno. ¿Verdadero o falso?

\section{Juego de dominó}

Actividad utilizada en la temática niveles de organización de los ecosistemas (fotografía 2).

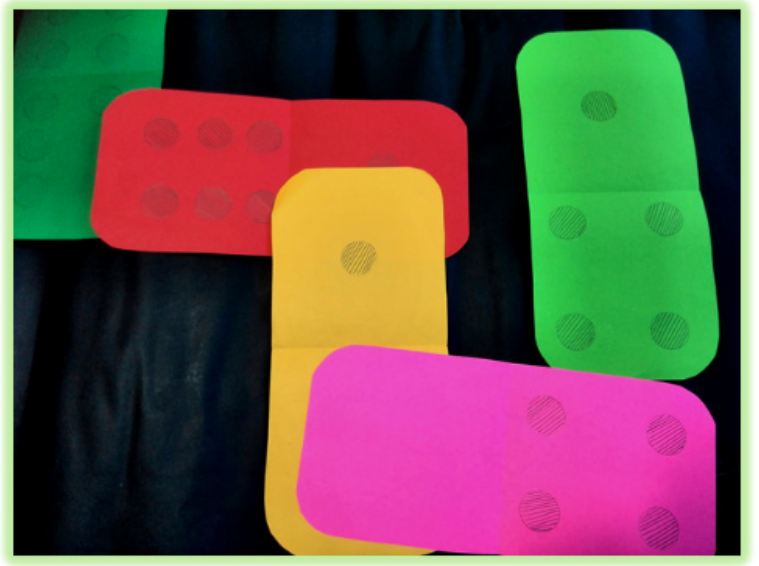

Fotografía 2. Fichas realizadas por la maestra.

En esta actividad, el grupo se dividió en dos, y cada estudiante tenía una o dos fichas del dominó. Dependiendo de la ficha que iba a utilizar, el estudiante que la tenía, respondía una pregunta, predeterminada por la maestra desde el principio de acuerdo a su valor (por ejemplo, para la ficha 6-6 había una pregunta y así para todos los valores). Por cada acierto se obtenía un punto. Ganaba el grupo que al final obtuviera mayor puntaje. 
Como ejemplo de las preguntas utilizadas se pueden citar:

- ¿Qué es una comunidad?

- Una población está conformada por organismos de diferentes especies. ¿Verdadero o falso?

- Los biomas están conformados por ecosistemas. ¿Verdadero o falso?

- ¿Cuál es el nivel de organización biológica que agrupa a los ecosistemas con características similares?

- ¿Cuál es el nombre que recibe el conjunto de organismos de una misma especie?

- Una comunidad biológica se compone de poblaciones de diferentes especies. ¿Verdadero o falso?

\section{Juego carreras de observación}

Actividad utilizada en la temática: redes y cadenas tróficas.

Para la carrera de observación, se organizaron grupos de tres estudiantes. Cada grupo elaboró tres preguntas y las ubicó en diferentes partes del colegio. Posteriormente, todos los grupos buscaban y respondían el total de las preguntas. La idea era que todos los grupos lograran encontrarlas y solucionarlas todas.

Como ejemplo de las preguntas utilizadas se pueden citar:

- En las cadenas tróficas se representa el flujo de materia y energía de manera lineal. ¿Verdadero o falso?

- ¿Qué necesitan los organismos para realizar las funciones vitales?

- Dé tres ejemplos de productores.

- Un consumidor se alimenta de más de una especie del nivel inferior y sirve de alimento a varios individuos del nivel superior. ¿Verdadero o falso?

- La presencia de los productores, consumidores y descomponedores en los ecosistemas hace posible que el flujo de la materia sea cíclico. ¿Verdadero o Falso?

\section{Tercera fase: evaluación de la utilidad del juego}

A lo largo de esta fase, para identificar la utilidad del juego y el progreso de los estudiantes en el manejo de los contenidos y de las temáticas desarrolladas, se realizaba una sustentación por cada temática.

Las sustentaciones constaban de diez preguntas: cinco de selección múltiple y cinco abiertas, en las cuales se evidenciaba el manejo de los temas. Al revisarlas se tenía en cuenta la descripción, el análisis y la relación de los conceptos.

\section{Cuarta fase: elaboración de la escala según el criterio de avance para verificar la utilidad del juego}

Se elaboró una escala que describe el criterio de avance según el número de aciertos en las preguntas y su respectiva valoración (tabla 1).

Tabla 1. Criterios de valoración para números de aciertos

\begin{tabular}{|c|c|c|}
\hline $\begin{array}{c}\text { N. }{ }^{\circ} \text { de } \\
\text { aciertos }\end{array}$ & Criterio & \multicolumn{1}{|c|}{ Valoración } \\
\hline $1-4$ & Bajo & $\begin{array}{l}\text { Muestra baja apropiación de los } \\
\text { temas trabajados. }\end{array}$ \\
\hline $\mathbf{5 - 7}$ & Medio & $\begin{array}{l}\text { Muestra un nivel básico. } \\
\text { Apropiación adecuada de las } \\
\text { temáticas trabajadas. }\end{array}$ \\
\hline $7-8$ & Alto & $\begin{array}{l}\text { Muestra una buena apropiación de } \\
\text { las temáticas trabajadas. }\end{array}$ \\
\hline $9-10$ & Muy alto & $\begin{array}{l}\text { Muestra una sobresaliente } \\
\text { apropiación de las temáticas } \\
\text { trabajadas. }\end{array}$ \\
\hline
\end{tabular}

Fuente: elaboración propia.

Para consolidar y contrastar los datos obtenidos, se promedió el número de estudiantes, según la cantidad de aciertos en las preguntas para las ocho sustentaciones, cuatro antes de los juegos y cuatro después de ellos.

\section{Quinta fase: encuesta a los estudiantes para conocer su percepción frente a los juegos}

Se realizó una encuesta a los estudiantes sobre los aportes del juego a su proceso de aprendizaje, de manera que se pudiera determinar su visión al respecto. La actividad se orientó con las siguientes preguntas:

- ¿Qué es el juego?

- ¿Cuáles son los aspectos positivos de los juegos desarrollados en la clase?

- ¿Cuáles son los aspectos por mejorar?

- ¿Considera que su desempeño académico mejoró al implementar estas actividades?

Para el análisis de las respuestas de los estudiantes, se codificaron por la letra E (Estudiante), acompañados de los números ordinales (E1, E2, E3, ... E41). Y se usó el método descriptivo-interpretativo, tratando de obtener el mínimo de detalle de lo que se está investigando (Rodríguez, Gil y García, 1996). 
Para comprobar la confiabilidad y validez de los hallazgos, se utilizaron cinco grupos de discusión con cuatro estudiantes cada uno. Esta es una técnica flexible y abierta, en la que cada individuo actúa en el grupo desde sus propios intereses y necesidades, expresando sus experiencias y vivencias desde su punto de vista personal. Este microcosmos reproduce las formas de operar de la sociedad y la cultura en la que se asientan dichos grupos (Zapata, 2005).

Una vez hecho esto, se organizó la información en categorías, según la grounded theory, o método comparativo constante (MCC), recurriendo al análisis descriptivo y el análisis relacional. En esta metodología se trabaja con categorías emergentes, maximizando las posibilidades de descubrir aspectos acerca del objeto de estudio; además, el material es fragmentado, y cada unidad de sentido se conceptualiza y nomina, es decir se le adscribe una "etiqueta verbal" (Araya, 2002) que permite interpretar el significado de la información, para luego agruparla en categorías que a su vez son evaluadas y sintetizadas en unas más grandes o de nivel superior.

A continuación, se presenta de manera general la estructura y los tiempos de clase empleados en las fases descritas. Por temática, se emplearon en total ocho horas, cada una de 45 minutos. Estas se encontraban divididas según el horario escolar por días, por ejemplo, dos horas el lunes, dos el martes y una el jueves.

\section{Primera fase}

- Durante las dos primeras horas la maestra explicaba el tema.

- Después, en las dos horas siguientes, se realizaban ejercicios prácticos. Por ejemplo:

» Para el flujo de materia y energía, los estudiantes de cada curso se dividieron en grupos de cuatro (cinco en total), para mostrar gráficamente en una cartelera uno de los ciclos trabajados (nitrógeno, carbono, oxígeno, agua, azufre).

»En las redes tróficas, los estudiantes presentaban ejemplos de una cadena y una red trófica consultada previamente.

»Con relación a los niveles de organización de los ecosistemas, nuevamente por grupos, los estudiantes diseñaban unas preguntas del tema, cuya respuesta se encontraba en una sopa de letras. Estas se intercambiaron por grupos.

- Al final de las actividades prácticas, en una hora, se realizaba una sustentación escrita con diez preguntas que recogían los aspectos trabajados.

\section{Segunda fase}

En las dos siguientes horas se implementaba el juego de preguntas.

\section{Tercera fase}

En una hora se realizaba una nueva sustentación de la temática.

Las sustentaciones se realizaron teniendo en cuenta que el estudiante aprende de y a partir de la propia evaluación y de la corrección, de la información contrastada que le ofrece el profesor, que será siempre crítica y argumentada, pero nunca descalificadora ni paralizadora (Álvarez, 2011). En ese sentido, la revisión de las respuestas se dio teniendo en cuenta que los estudiantes presentaran un buen dominio de los contenidos y temáticas desarrolladas.

\section{Cuarta fase}

En esta fase se elaboró la escala de valoración, por lo que no se requirió de espacios de clase adicionales con los estudiantes.

\section{Quinta fase}

Para llevar a cabo la encuesta, se empleó una hora de clase una vez se implementó la fase cuatro.

\section{Resultados}

En las tablas 2 y 3 se presentan los datos promediados de los aciertos de las respuestas de los estudiantes en las sustentaciones antes y después de realizar los juegos.

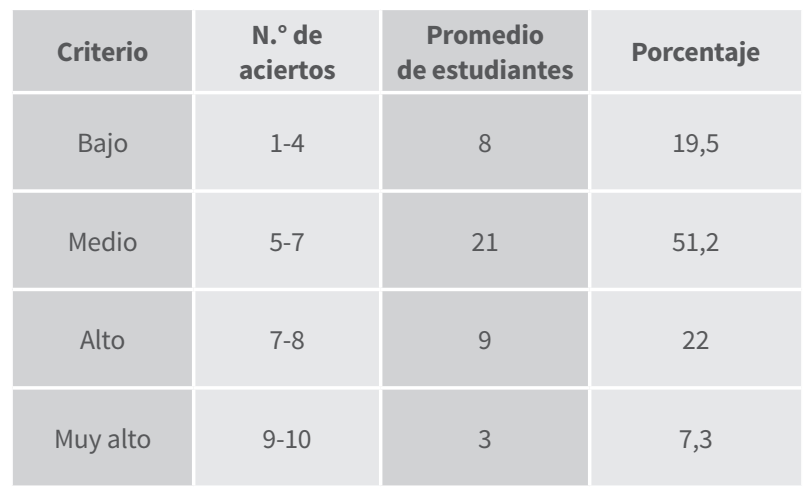

Fuente: elaboración propia. 
Tabla 3. Criterios de valoración por números de aciertos en las cuatro sustentaciones después de los juegos

\begin{tabular}{|c|c|c|c|}
\hline Criterio & $\begin{array}{c}\mathbf{N} \mathbf{*}^{\circ} \mathbf{d e} \\
\text { aciertos }\end{array}$ & $\begin{array}{c}\text { Promedio } \\
\text { de estudiantes }\end{array}$ & Porcentaje \\
\hline Bajo & $1-4$ & 0 & 0 \\
\hline Medio & $5-7$ & 16 & 39 \\
\hline Alto & $7-8$ & 18 & 44 \\
\hline Muy alto & $9-10$ & 7 & 17 \\
\hline
\end{tabular}

Fuente: elaboración propia.

Para facilitar los contrastes de los resultados antes y después de los juegos de preguntas, se presenta la gráfica 1.

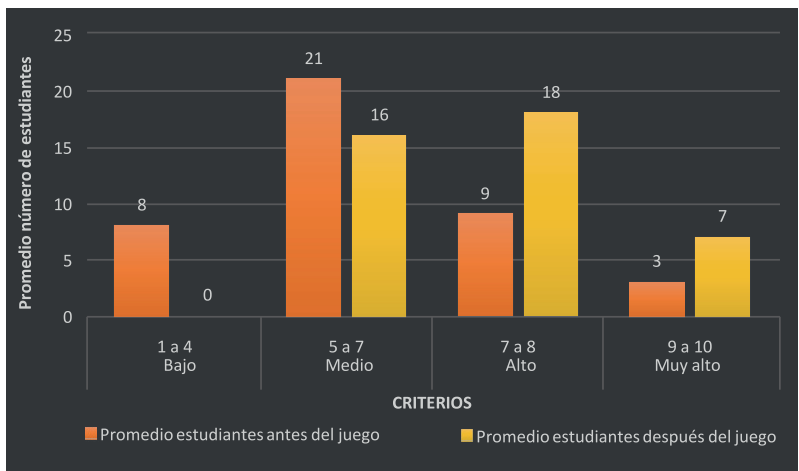

Gráfica 1. Valoración por número de aciertos en las cuatro sustentaciones antes y después de los juegos.

Fuente: elaboración propia.

\section{Resultados de la encuesta}

\section{Para la pregunta ¿Qué es el juego?}

El $100 \%$ de los estudiantes estableció el juego como una actividad de refuerzo dentro del contexto académico. Sin embargo, a partir de sus explicaciones se establecieron 5 subcategorías (tabla 4).
Tabla 4. El juego visto como actividad de refuerzo en el contexto académico

\begin{tabular}{|c|c|c|}
\hline Categoría & Subcategoría & Comentario \\
\hline \multirow{5}{*}{$\begin{array}{l}\text { Actividad } \\
\text { académica }\end{array}$} & $\begin{array}{l}\text { Desarrollo físico y } \\
\text { mental }\end{array}$ & $\begin{array}{l}\text { "Es una actividad mental o } \\
\text { física en la cual participamos y } \\
\text { nos ayuda a entender" E16 }\end{array}$ \\
\hline & Entretenimiento & $\begin{array}{l}\text { "Una actividad de } \\
\text { entretenimiento para los } \\
\text { estudiantes, que nos permite } \\
\text { entender más" E12 } \\
\text { "Es una actividad que } \\
\text { entretiene y en que se está } \\
\text { concentrado y se aprende" E7 } \\
\text { "Actividad que es placentera } \\
\text { y donde no sentimos ninguna } \\
\text { presión" E6 } \\
\text { "Es una alternativa de } \\
\text { aprendizaje que llama más la } \\
\text { atención de los estudiantes" } \\
\text { E26 } \\
\text { "Actividades que nos } \\
\text { entretienen mientras } \\
\text { aprendemos "E11 }\end{array}$ \\
\hline & $\begin{array}{l}\text { Como proceso de } \\
\text { autoevaluación }\end{array}$ & $\begin{array}{l}\text { "Es una forma interactiva y } \\
\text { entretenida de mostrar cómo } \\
\text { va nuestra comprensión de lo } \\
\text { visto en clase" E28 }\end{array}$ \\
\hline & $\begin{array}{l}\text { Desarrollo de } \\
\text { contenidos }\end{array}$ & $\begin{array}{l}\text { "Es una actividad de repaso" } \\
\text { "Es una manera de llevar a } \\
\text { cabo los distintos temas" E18 } \\
\text { "Es una manera más didáctica } \\
\text { de aprender y entender los } \\
\text { conceptos en clase" E15 }\end{array}$ \\
\hline & $\begin{array}{l}\text { Mecanismo de } \\
\text { participación }\end{array}$ & $\begin{array}{l}\text { "Actividades que nos permiten } \\
\text { participar más" E9 }\end{array}$ \\
\hline
\end{tabular}

Fuente: elaboración propia. 
Para la pregunta ¿cuáles son los aspectos positivos de los juegos desarrollados en la clase?

Se establecieron cinco categorías (tabla 5).

Tabla 5. Aspectos positivos de los juegos desarrollados en la clase

\begin{tabular}{|c|c|}
\hline Categoría & Comentario \\
\hline $\begin{array}{l}\text { Cambios de } \\
\text { dinámica en la } \\
\text { clase }\end{array}$ & $\begin{array}{l}\text { "Los juegos permiten salir de la rutina en la } \\
\text { clase" E8 } \\
\text { "Las clases son más entretenidas" E1 }\end{array}$ \\
\hline $\begin{array}{l}\text { Involucra a los } \\
\text { estudiantes }\end{array}$ & $\begin{array}{l}\text { "Al involucrar a los estudiantes estos se } \\
\text { vuelven más activos y se divierten más" E15 } \\
\text { "Nos unimos más como grupo, nos permite } \\
\text { ayudarnos entre sí" E27 } \\
\text { "Permite reconocer las fortalezas de los otros" } \\
\text { E41 } \\
\text { "Nos ayudamos como grupo para que no } \\
\text { haya dudas o problemas para comprender } \\
\text { temas más adelante" E29 }\end{array}$ \\
\hline Reto personal & $\begin{array}{l}\text { "Todos son juegos de cierta forma } \\
\text { competitivos, pero más para competir con } \\
\text { uno mismo, lo que es un incentivo para } \\
\text { realizarlo lo mejor posible". E6 } \\
\text { "Cuando los juegos son de competencia } \\
\text { los estudiantes se ven más emocionados a } \\
\text { completar las actividades" E38 } \\
\text { "Permite que los estudiantes vean que } \\
\text { aprender no tiene que ser aburrido" E32 }\end{array}$ \\
\hline $\begin{array}{l}\text { Consolida el } \\
\text { trabajo en grupo }\end{array}$ & $\begin{array}{l}\text { "Fomenta espacios para socializar como } \\
\text { grupo" E3 } \\
\text { "La actividad permite tener ese sentimiento } \\
\text { de satisfacción en ti y el grupo" E24 }\end{array}$ \\
\hline
\end{tabular}

Fuente: elaboración propia.

\section{Para la pregunta: ¿cuáles son los aspectos por mejorar?}

El $100 \%$ de los estudiantes coincidió en que la falta de compromiso en los repasos no permitió aprovechar al máximo las actividades. Para ilustrar este punto se presentan dos comentarios:

"Estos juegos aunque nos ayudan mucho, la mayoría de las veces el mayor problema para su correcta elaboración es la falta de compromiso de muchos de nosotros que no repasamos" E19

"Hace falta que haya mayor compromiso del jugador y del compañero que lo organiza" E2

Para la pregunta ¿Considera que su desempeño académico mejoró al implementar estos juegos?
Todos los estudiantes coincidieron en la mejoría de su proceso, porque contribuyó a generar actitudes positivas. A partir de sus respuestas se presentan cinco categorías (tabla 6).

Tabla 6. Aspectos que mejoran el desempeño académico mediante el juego

\begin{tabular}{|c|c|}
\hline Categoría & Comentario \\
\hline Cooperación & $\begin{array}{l}\text { "Como son en equipo, los compañeros } \\
\text { suelen ayudarme a entender" E23 } \\
\text { "Si alguien se equivoca, nos explicamos } \\
\text { por qué esa no es la respuesta y esto ayuda } \\
\text { a asimilar". E18 }\end{array}$ \\
\hline Mayor compromiso & $\begin{array}{l}\text { "Cuando hay actividad procuro estudiar } \\
\text { más" E22 } \\
\text { "Me ha ayudado a realizar las actividades } \\
\text { de repaso en casa" } E 13 \\
\text { "Las actividades son de mi gusto y me } \\
\text { comprometo a estudiar" E41 } \\
\text { "Hacer juegos y jugarlos me motiva más } \\
\text { para responder y me esfuerzo más por } \\
\text { expresar las preguntas" E3 }\end{array}$ \\
\hline $\begin{array}{l}\text { Identificar aspectos a } \\
\text { reforzar }\end{array}$ & $\begin{array}{l}\text { "Me doy cuenta de lo que me falta reforzar" } \\
\text { E30 } \\
\text { "Con las actividades puedo acordarme más } \\
\text { fácil y puedo relacionar más cosas" E15 } \\
\text { "Los juegos permiten que repase } \\
\text { más, entienda mejor y aclare dudas } \\
\text { y al momento de la sustentación las } \\
\text { calificaciones se vean afectadas de manera } \\
\text { positiva" E39 }\end{array}$ \\
\hline Mejorar habilidades & $\begin{array}{l}\text { "Mi nivel de concentración ha aumentado, } \\
\text { porque me interesa lo que hago, he } \\
\text { entendido los temas más fácilmente" E31 } \\
\text { "En mi proceso veo mucha mejoría, en } \\
\text { cuestiones de entendimiento y como } \\
\text { me expreso con palabras que antes no } \\
\text { entendía" E29 }\end{array}$ \\
\hline $\begin{array}{l}\text { Aumento de interés y } \\
\text { motivación }\end{array}$ & $\begin{array}{l}\text { "Ha mejorado el interés al tema y la clase y } \\
\text { esto hace que aprenda más" E21 } \\
\text { "La clase es más interesante y me distraigo } \\
\text { menos lo cual ayuda a que entienda mejor" } \\
\text { E36 } \\
\text { "Me han ayudado pues me divierto } \\
\text { aprendiendo" E11 }\end{array}$ \\
\hline
\end{tabular}

Fuente: elaboración propia.

\section{Discusión}

Como puede verse en la gráfica 1 , las respuestas agrupadas por criterios y puntuación permiten identificar el rango de desempeño de los estudiantes antes y después de realizar las actividades. 
Así, los datos evidencian que los estudiantes mejoraron considerablemente después de los juegos de preguntas, ya que en la primera parte (tabla 2) se encuentra un $19,5 \%$ dentro del criterio Bajo, con un número de aciertos entre uno y cuatro. Pero después de las actividades (tabla 3 ) no hay estudiantes dentro de este criterio. Además, el nivel Medio disminuyó en un 12,2 \%, mientras que el Alto aumentó un $22 \%$, es decir, el doble de los estudiantes se encontraba dentro de este criterio.

Asimismo, después de los juegos de preguntas, se pasó de un $7,3 \%$ en el criterio Muy alto, a un $17 \%$ con un número de aciertos entre 9 y 10 preguntas.

Estos resultados están relacionados con ver los juegos de preguntas como actividades agradables que mueven sus expectativas y facilitan su acercamiento al contexto académico, en la medida en que permiten mejorar en los aspectos físico y mental, son entretenidas, y que permiten el desarrollo de contenidos y procesos de autoevaluación, como se muestra en la tabla 4.

En este sentido, como lo menciona Bruner (1983), el juego es un ejercicio que permite suavizar la tarea desempeñada por el joven, a la vez que contribuye a la memorización como factor de aprendizaje, puesto que la memoria corresponde a los cambios que se producen en el cerebro (Morgado y Bernal, 2014) para retener o almacenar lo que aprendemos. De esta manera, aprendizaje y memoria son dos fenómenos interdependientes, ya que el aprendizaje implica adquisición de información y por lo tanto una modificación del estado de memoria del sujeto (Aguado, 2001).

En consecuencia, a medida que se desarrollan los juegos de preguntas, el estudiante tiene la posibilidad de crear una confrontación entre lo que conoce y lo que responde su compañero (respuesta E28, tabla 4), todo esto comparado a su vez con la retroalimentación realizada por parte del maestro.

De igual manera, las actividades que involucran a los estudiantes activamente en el proceso de enseñanza-aprendizaje facilitan que se desarrollen factores indispensables en su desarrollo, como son los aspectos psíquicos, intelectuales y sociales (Prieto 1984, citado en Euceda, 2007). Estos se evidencian en las categorías Involucra a los estudiantes y Reto personal, presentadas en la tabla 5.

Por lo anterior, puede decirse que los juegos de preguntas sirvieron de enlace a los contenidos conceptuales, procedimentales y actitudinales, como se observa en la tabla 6. Allí se resaltan: la cooperación, mayor compromiso con la temática y las clases, la identificación por parte de los estudiantes de los temas a reforzar, de igual manera el aumento de la motivación, lo que los lleva a centrar su interés por el aprendizaje. En este sentido, según Csikszentmihalyi (1998) para que el estudiante pueda mantener el interés en una materia tiene que disfrutar trabajando con ella.

\section{Conclusiones}

\section{Antes de introducir los juegos}

- Para llevar a cabo diferentes actividades de juego, el maestro debe tener claro cuáles son sus objetivos y evaluar de qué manera estos juegos responden a esos objetivos. Para ello puede evaluar las finalidades pedagógicas propuestas por Dogbeh y N'Diaye, (1980).

- El maestro tiene el reto de plantear, diseñar e implementar diversas formas de enseñanza dentro del aula, para posibilitar la mejora continua del proceso de enseñanza-aprendizaje.

- El maestro ha de reconocer el juego no solo como un espacio lúdico, sino como un espacio de desarrollo cognitivo y social. Para eso es necesario considerar que educar creativamente es educar para el cambio y capacitar para la innovación como lo sugieren De la Torre y Marín (1991).

\section{Con relación a la importancia del juego en el aula}

- El juego es un elemento esencial en la vida del ser humano, que afecta de manera diferente cada periodo de la vida, y que por sus posibilidades tienen un gran valor para la educación (Prieto, 1984). Por ende, se cuenta con los juegos didácticos o educativos que están elaborados para facilitar el ejercicio de las funciones mentales en general.

- El juego en el aula es una excelente estrategia didáctica, como lo menciona Martínez (2010) ya que en el juego el niño interactúa con el medio que lo rodea, se producen experiencias de interacción social, que permiten que los estudiantes sean responsables de sus decisiones y afronten sus consecuencias. Esto consolida su autonomía.

- Los juegos planificados correctamente facilitan la integración de los contenidos de las diversas áreas y los entrelazan de manera tranquila y placentera.

\section{Con relación a los juegos de preguntas, en la enseñanza de las ciencias}

- Los juegos de preguntas ayudan a mejorar la memoria y la atención en los estudiantes, aspectos que contribuyen al reconocimiento de las temáticas y desde allí se facilita su posible articulación y relación. 
- El uso del juego incentiva el desarrollo moral, al permitir el reconocimiento del otro (de los pares y el maestro) dentro de una serie de normas y acuerdos. Además, contribuye al desarrollo social al respetar y compartir otros puntos de vista.

- Los juegos de preguntas como elemento principal en las estrategias para facilitar el proceso de enseñanza-aprendizaje deben ser actividades agradables que le permitan al estudiante vincular de manera amena las diferentes temáticas trabajadas.

- Los juego de preguntas deben tener reglas claras desde el principio, para que se fortalezcan la construcción de valores como el respeto, la comunicación y la colaboración entre otros.

\section{Aportes del trabajo en el grupo}

- Permitió a los estudiantes ver los errores como una posibilidad de poner a prueba los conocimientos y así encontrar las soluciones a los problemas.

- El juego propició la motivación por el área. Al ser la motivación la fuerza interna que dinamiza al individuo en dirección a una meta y a unos resultados específicos, originada en una necesidad, carencia o alteración del bienestar, ya sea por exceso o por defecto (Montero, 2009).

- Incluir estos juegos en el aula permitió en los alumnos la creación de hábitos de estudio, a través de un constante repaso, lo que les permitió recordar y relacionar cada vez más los temas abordados.

- Permite conocer un poco más a los estudiantes, observando sus fortalezas y debilidades no solo desde lo académico sino también desde lo social, de manera que se puede orientar el proceso desde lo individual y lo colectivo.

- Los juegos de preguntas les sirvieron a los estudiantes como un medio de enlace a los contenidos conceptuales, procedimentales y actitudinales.

Como producto de la actividad investigativa, se desprende que:

- La puesta en marcha de esta propuesta permitió evidenciar que implementar estrategias lúdicas en el aula genera beneficios que pasan por lo afectivo, lo cognoscitivo y lo comportamental. Por lo tanto, en la medida en que se realicen con más regularidad en el proceso de enseñanza-aprendizaje permitirá acercar a los estudiantes a la comprensión de los conceptos, además hará más amena la clase tanto para los estudiantes como para los maestros.

- Si bien los juegos empleados en esta investigación tradicionalmente se usan en otros espacios fuera de la clase, y no estuvieron diseñados para involucrar acti- vamente los conceptos, demuestran que durante las clases se puede trabajar la programación establecida (desde los contenidos conceptuales, procedimentales y actitudinales) de diferentes maneras, logrando vincular aún más a los estudiantes en su proceso de formación.

- Los análisis de resultados ponen de manifiesto que los estudiantes opinan que el juego los atrae y motiva a aprender. Por eso se constituye en un poderoso recurso metodológico.

\section{Agradecimientos}

A todos los niños y niñas del grado séptimo, cursos A y $B$, por su buen ánimo y disposición en cada una de las actividades.

\section{Referencias}

Afanador, H. y Mosquera, C. (2017). Valoración de actitudes hacia la ciencia y actitudes hacia el aprendizaje de la biología en educación secundaria (pp. 32-49). Bio-Grafía, 5(8), 32-49. Recuperado de https://doi.org/10.17227/20271034. vol.5num.8bio-grafia32.49.

Aguado, A. L. (2001). Aprendizaje y memoria. Revista de Neurología, 32(4), 373-381.

Alonso, J. (1998). Motivación y aprendizaje en el aula: cómo enseñar a pensar. Madrid: Santillana.

Álvarez, M. J. (2011). Evaluar para conocer examinar para excluir. Madrid: Morata.

Araya. U. S (2002). Las representaciones sociales. Ejes teóricos para su discusión. Costa Rica: Facultad Latinoamericana de Ciencias Sociales (Flacso).

Becerra, Á., Valderrama, W., y Torres, N. (2014). Las percepciones de los niños de primaria acerca de las especies carismáticas y no carismáticas. Bio-Grafía, 361-367. Recuperado de https://doi.org/10.17227/20271034. vol.0num.0bio-grafia361.367

Blanchard, K. y Chesca, A. (1986). Antropología del deporte. Barcelona: Bellaterra.

Bruner, J. (1983). Jugar, juegos y lenguaje. En el habla en el niño. Barcelona: Paidós.

Burton, S. y Dobson, C. (2009). Spork \& beans: Addressing evolutionary misconceptions. The American Biology Teacher, 71(2), 89.

Cagigal, J. M. (1971). Necesidad pedagógica del ocio activo en el hombre de nuestro tiempo. Madrid: INEF. 
Caillois, R. (1986). Los juegos y los hombres. Las máscaras y el vértigo. México: Fondo de Cultura Económica.

Cerda, H. (2002). Elementos de la investigación como reconocerlos, diseñarlos y construirlos (4. ${ }^{\mathrm{a}} \mathrm{ed}$.). Quito: Abya Yala.

Cook, D. A. y Artino, A. R. (2016). Motivation to learn: An overview of contemporary theories. Medical Education, 50(10), 997-1014. Recuperado de http:// doi.org/10.1111/medu.13074.

Csikszentmihalyi, M. (1998). Creatividad. El fluir y la psicología del descubrimiento y la invención. Barcelona: Paidós.

De la Torre. S. y Marín, R. (Coord.). (1991). Manual de la creatividad. Aplicaciones educativas. Barcelona: Vicens Vives.

Deveau, J., Jaeggi, M., Zordan, V., Phung, C. y Seitz, A. (2014). Cómo construir mejores juegos de entrenamiento de memoria. Frontiers in Systems Neuroscience. 8, 243. Recuperado de http://doi. org/10.3389/fnsys.2014.00243.

Díaz, B. A. (1999). Didáctica y currículum. Convergencia en los programas de estudio. México: Paidós Educador.

Dogbeh, R. y N'Diaye, S. (1980). Use of games and toys for educational purposes Unesco: documento inédito.

Erikson, E. (1972). Juego y actualidad. En: J. Piaget, y K. Lorenz, Juego y desarrollo. Barcelona: Grijalbo.

Escudero, J. M. (1981): Modelos didácticos. Barcelona: Oikos-Tau.

Estebaranz, A. (1994). Didáctica e innovación curricular. Sevilla: Publicaciones de la Universidad de Sevilla.

Euceda, T. M. (2007). El juego desde el punto de vista didáctico a nivel de educación prebásica (tesis de maestría). Universidad Pedagógica Nacional Francisco Morazán. Tegucigalpa.

Fernández, H. J. (1974): Didáctica. Madrid: UNED.

Gargallo, B., Pérez, C., Serra, B., Sánchez, F. y Ros, C. (2007). Actitudes ante el aprendizaje y rendimiento académico en los estudiantes universitarios. Revista Iberoamericana de Educación, 42, 1-25.

Giordan, A. (1989). De las concepciones de los alumnos a un modelo de aprendizaje alostérico. Investigación en la Escuela, 8, 3-14.

Goldstone, R. L. (1998). Perceptual learning. Ann. Rev. Psychol., 49, 585-612.
González, D. y Maytorena, M. (2001). Influencia de la orientación motivacional, los estilos de aprendizaje y los factores de carrera sobre el desempeño escolar. Revista Mexicana de Psicología, 18(número especial 1$)$.

Gruppe, O. (1976). Teoría pedagógica de la educación física. Madrid: INEF.

Hernández, R., Fernández, C. y Baptista, P. (2003). Metodología de la investigación ( $3^{\mathrm{a}}$ ed.). México: McGraw-Hill.

Jiménez, H. y Hernández, J. C. (2001). ¿Cómo motivar a los alumnos para que aprendan?: la motivación en la enseñanza. En: A. Rodríguez (coord.). Psicología de la Instrucción para Enseñantes (pp. 199-227). Instituto Psicosocial Manuel Alemán Álamo, Las Palmas de Gran Canaria.

Kafai, Y. B. y Burke, Q. (2015). Juego constructivista: comprender los beneficios de crear juegos para el aprendizaje. Psicólogo Educacional, 50(4), 313-334. Recuperado de http://doi.org/10.1080/00461520.2 015.1124022 .

Klein, M. (1929). La personificación en el juego de los niños. Buenos Aires: Hormé.

Latham II, L. G., y Scully, E. P. (2008). Critters! A realistic simulation for teaching evolutionary biology. The American Biology Teacher, 70(1), 30.

Mallart, J. (2001). Didáctica: Concepto, objeto y finalidades. En: F. Sepúlveda y N. Rajadell (coords.) Didáctica General para Psicopedagogos. Madrid: UNED.

Martínez Quesada, M. (2010). El juego como método de aprendizaje. Revista Digital Enfoques Educativos, $71,102-112$.

Mayr, E. (2006). Por qué es única la biología. Buenos Aires: Katz Editores.

Melo Herrera, M. P. y Hernández Barbosa, R. (2014). El juego y sus posibilidades en la enseñanza de las ciencias naturales. Innovación Educativa, 14(66), 41-64.

Melo H., M. (2017). Construcción de juegos de mesa como recurso didáctico para promover el desarrollo de habilidades comunicativas en ciencias. Bio-Grafía, 10(18), 124-131. Recuperado de https://doi.org/10.17227/20271034. vol.10num.18bio-grafia124.131.

Mengascini, A. y Menegaz, A. (2005). El juego de las mariposas. Propuesta didáctica para el tratamiento del cambio biológico. Eureka sobre Enseñanza y Divulgación de las Ciencias, 2(3), 403-415. 
Montero, M. L. (2009). El rendimiento académico y la motivación en las aulas de Educación Primaria. Andalucía Educa, 8, 84-85.

Moor, P. (1981). El juego en la educación. Barcelona: Herder.

Morgado, I. y Bernal I. M. (2014). Aprender, recordary olvidar. Barcelona: Ariel.

Muñoz de Bustillo, M. C., Hernández, P. y García, L. A. (1998). Mejorando el clima del aula. Evaluación e Intervención Psicoeducativa, 1, 169-214.

Newman, F. (1992). Student engagement and achievement in American secondary schools. Nueva York: Teachers College Press.

Odum, E. (1971). Fundamentals of ecology (3. ${ }^{\text {a }}$ ed.). Filadelfia, Estados Unidos: W. B. Saunders.

Orozco Marín, Y. y Perdomo Gómez, J. (2015). El juego como herramienta para la enseñanza del funcionamiento del sistema nervioso en los seres vivos y aporte a la solución de problemas de convivencia en el aula. Bio-Grafía, número extraordinario. 1389-1399. Recuperado de https://doi.org/10.17227/20271034. vol.0num.0bio-grafia1389.1399.

Oviedo, G. L. (2004). La definición del concepto de percepción en psicología con base en la teoría Gestalt. Revista de Estudios Sociales, 18, 89-96. Recuperado de http://www.scielo.org. co/scielo.php?script=sci_arttext \&pid=S0123 $885 \times 2004000200010 \& \operatorname{lng}=e n \& \operatorname{lng}=e s$.

Piaget, J. (1971). El criterio moral en el niño. Barcelona: Fontanella.

Prieto Figueroa, L. (1984). Principios generales de la educación. Caracas: Monte Ávila.
Reeve, J. (1996). Motivación y emoción. Madrid: McGraw-Hill.

Rodríguez, G., Gil, Javier y García, E. (1996). Metodología de la investigación cualitativa. Málaga, España: Aljibe.

Ryan, R. M. y Deci, E. L. (2000). Intrinsic and extrinsic motivations: Classic definitions and new directions. Contemporary Educational Psychology.

Sanabria, I., Sandoval, L. y Arango, A. (2017). El juego como estrategia para la enseñanza y el aprendizaje de la evolución biológica en estudiantes de noveno grado. Bio-Grafía, 10(19), 146-152. Recuperado de https://doi.org/10.17227/bio-grafia. extra2017-7092.

Santisteban Cimarro, A. (1990). El uso de juegos de simulación en ciencias naturales como técnicas de conocimiento del entorno. Investigación en la Escuela, 10, 71-75.

Stenhouse, L. (1987). La investigación como base de la enseñanza. Madrid: Morata.

Stewart, J. H. (1983). Student problem solving in high school genetics. Science Education, 4, 523-540.

Titone, R. (1976): Metodología didáctica. Madrid: Rialp.

Wallon, H. (1942). El juego en la evolución psicológica del niño. Buenos Aires: Psique.

Winnicott, D. W. (1971). Realidad y juego. Barcelona: Gedisa.

Zapata. A. O. (2005). Herramientas para elaborar investigaciones socioeducativas. México: Pax. 


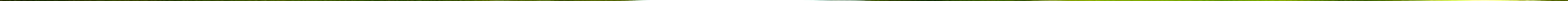

